\title{
Controle por Regras: Efeitos de Perguntas, Propriedades Formais de Regras e Esquemas de Reforço
}

\author{
Control by Rules: Effects of Questions, Formal Properties \\ of Rules and Reinforcement Schedules
}

\author{
Adelina Santana Gonçalves*, Luiz Carlos de Albuquerque \& Carla Cristina Paiva Paracampo \\ Universidade Federal do Pará, Belém, PA, Brasil
}

\begin{abstract}
Resumo
Com o objetivo de investigar os efeitos de variáveis sobre o seguimento de regras, 24 estudantes universitários foram expostos a um procedimento de escolha de acordo com o modelo. A tarefa era apontar para os estímulos de comparação em sequência. Na Fase 1, a sequência correta era estabelecida por reforço diferencial em esquema de reforço contínuo. Nas Fases 2 e 3, as contingências de reforço eram mantidas inalteradas, enquanto as regras discrepantes de tais contingências (sugestão e ordem) eram manipuladas. Nas Condições 1 e 2, nas Fases 2 e 3, eram apresentadas a sugestão e a ordem, respectivamente. Nas Condições 3 e 4, era o inverso. Apenas na Fase 1 das Condições 1 e 3 eram feitas perguntas acerca do comportamento que produz reforço. $100 \%$ dos participantes não seguiram a sugestão e $60 \%$ não seguiram a ordem. Os resultados sugerem que a história do comportamento alternativo ao especificado pela regra, justificativas e perguntas são variáveis que podem interferir no comportamento de seguir regras discrepantes das contingências de reforço. Os resultados têm implicações para o esclarecimento das funções das contingências de reforço e de regras na explicação do comportamento.

Palavras-chave: Regras e contingências, justificativas, histórias experimentais, perguntas.
\end{abstract}

\begin{abstract}
Aiming the investigation of the effects of some variables on rule following, 24 college students were submitted to a matching-to-sample procedure, with the task of pointing to the comparison stimuli in sequence. In Phase 1 the correct sequence was established by differential reinforcement in continuous reinforcement schedule. In Phases 2 and 3 the reinforcement contingencies were kept unchanged, and meanwhile rules (suggestion and mand) discrepant of the contingencies were manipulated. In Conditions 1 and 2, Phases 2 and 3 suggestion and mand were presented in this sequence. In Conditions 3 and 4 the presentation order was reversed. Questions concerning why the behavior was reinforced were presented only in Phase 1 of Conditions 1 and 3. Hundred percent participants did not follow the suggestion, $60 \%$ did not follow the mand. Results indicate that the history of alternative behavior to the specified by the rule, justifications, and questions may interfere with the discrepant-to-the-contingencies rule-following, and implications for the comprehension of the functions of the reinforcement contingencies and rules in the explanation of behavior.

Keywords: Rules and contingencies, justifications, experimental histories, questions.
\end{abstract}

Regras são estímulos antecedentes verbais que podem descrever o comportamento e suas variáveis de controle; estabelecer a topografia de comportamentos novos; e, alterar as funções de estímulos, independentemente das consequências imediatas produzidas pelo comportamento e de contiguidade espaço-temporal entre estímulo-comportamento e estímulo-estímulo (Albuquerque, Paracampo, Matsuo, \& Mescouto, 2013). Essa definição tem várias vantagens. Por exemplo, ela identifica as propriedades

* Endereço para correspondência: Universidade Federal do Pará, Núcleo de Teoria e Pesquisa do Comportamento, Rua Augusto Correa, 01, Guamá, Belém, PA, Brasil 66075-110. E-mail: adelinasantana@gmail.com, lcalbu@ ufpa.bre cparacampo@gmail.com definidoras de regras e está de acordo com as principais definições de regras da área (Schlinger \& Blakely, 1987; Skinner, 1969; Zettle \& Hayes, 1982). Em adição, ela considera que regras podem exercer múltiplas funções e, portanto, não deveriam ser definidas com base apenas em um de seus efeitos (Albuquerque, 2001).

De modo geral, os estudos na área de pesquisa do controle por regras têm sugerido que o comportamento governado por regras depende, em parte, do tipo de consequência imediata produzida pelo seguimento e pelo não seguimento de regra (Baron \& Galizio, 1983; Galizio, 1979; LeFrancois, Chase, \& Joyce, 1988; Paracampo \& Albuquerque, 2004; Paracampo, Albuquerque, Farias, Carvalló, \& Pinto, 2007); do tipo de esquema que reforça 
o seguimento e o não seguimento de regra (Newman, Buffington, \& Hemmes, 1995; Oliveira \& Albuquerque, 2007); da história do comportamento alternativo ao especificado pela regra (Albuquerque, de Souza, Matos, \& Paracampo, 2003; Albuquerque, Reis, \& Paracampo, 2006; Albuquerque, Silva, \& Paracampo, 2014; Oliveira $\&$ Albuquerque, 2007); da história de reforço diferencial para seguir e de punição para não seguir regras (Catania, Shimoff, \& Matthews, 1989; Cerutti, 1989; Chase \& Danforth, 1991; Hayes, Brownstein, Zettle, Rosenfarb, \& Korn, 1986; Paracampo, Souza, \& Albuquerque, 2014; Torgrud \& Holborn, 1990; Wulfert, Greenway, Farkas, Hayes, \& Dougher, 1994); e, da história de exposição a justificativas diferenciais para seguir e para não seguir regras (Albuquerque, 2005; Albuquerque \& Paracampo, 2010; Albuquerque et al., 2013; Albuquerque et al., 2014).

Outra importante variável que pode determinar o seguimento e o não seguimento de regras são as propriedades formais da própria regra (Albuquerque, 2005; Albuquerque, Mescouto, \& Paracampo, 2011; Albuquerque \& Paracampo, 2010; Braga, Albuquerque, Paracampo, \& Santos, 2010; Paracampo, Albuquerque, Mescouto, \& Farias, 2013). Propriedades formais de regras são as características apresentadas pela regra que determinam, em parte, o que ela parece ser ou o que ela indica em uma determinada comunidade verbal, de acordo com as suas práticas (Albuquerque \& Paracampo, 2010).

Por exemplo, Albuquerque et al. (2011) procuraram investigar os efeitos de perguntas acerca das contingências de reforço e de manipulações das propriedades formais das regras sobre o seguimento de regras. Para tanto, 24 estudantes universitários foram expostos a um procedimento de escolha de acordo com o modelo. Em cada tentativa, um estímulo modelo e três de comparação eram apresentados ao participante, que deveria apontar para os estímulos de comparação em sequência. Cada estímulo de comparação apresentava apenas uma dimensão - cor (C), espessura $(\mathrm{E})$ ou forma $(\mathrm{F})$ - em comum com o modelo e diferia nas demais.

Os estudantes foram distribuídos em quatro condições. Em cada condição, na Fase 1, o comportamento alternativo ao especificado pela regra (a sequência CEF) era modelado e depois mantido em esquema razão fixa 4 (FR 4). Nesse esquema razão fixa cada quatro emissões consecutivas da sequência correta produzia um ponto no contador. A não emissão consecutiva da sequência correta reiniciava a razão fixa - 4 para obtenção de um ponto. Nas Fases 2 e 3, as contingências eram mantidas inalteradas (isto é, a sequência $\mathrm{CEF}$ continuava sendo a única reforçada em FR 4), enquanto as propriedades formais das regras eram manipuladas (no caso, eram comparados os efeitos de uma regra discrepante com a forma de ordem com os efeitos de uma regra discrepante com a forma de sugestão).

Essas duas regras diferiam nos seguintes aspectos: A sugestão especificava: "Quando eu mostrar estes objetos para você, faça o que achar melhor para você. Se você quiser, você pode fazer o seguinte:”. Em seguida, especificava a sequência FCE e dizia que se o participante respondesse nessa sequência ele ganharia pontos. A ordem especificava: "Quando eu mostrar estes objetos para você, eu quero que você faça o seguinte:”. Em seguida, especificava a sequência ECF e dizia que se o participante respondesse nessa sequência ele ganharia pontos. Como já descrito, nos dois casos, o seguimento de regra não produzia ponto.

Na Fase 1 das Condições 1 e 3, eram feitas perguntas sobre qual a sequência correta e quantas vezes o participante tinha que apontar, consecutivamente, na sequência correta para ganhar um ponto. Nas Condições 2 e 4, não eram feitas perguntas. Nas Condições 1 e 2, a Fase 2 era iniciada com a sugestão e a Fase 3, com a ordem. Nas Condições 3 e 4, era o inverso.

Dos 24 estudantes, 2 ( 1 da Condição 3 e 1 da Condição 4) não aprenderam a sequência correta na Fase 1 e não foram expostos às Fases 2 e 3. Na Condição 1, 5 dos 6 participantes não seguiram a sugestão na Fase 2. Em contraste, na Condição 4 os cinco participantes seguiram a ordem na Fase 2. Nas Condições 2 e 3 houve variabilidade nos resultados. Ou seja, na Condição 2, 3 participantes seguiram e 3 deixaram de seguir a sugestão na Fase 2. Na Condição 3, 3 dos 5 participantes seguiram a ordem na Fase 2. Os resultados da Fase 2 das Condições 1 e 4 sugerem que: (a) o seguimento de regras discrepantes tem maior probabilidade de ser mantido quando a regra é apresentada na forma de uma ordem e o participante não é solicitado a responder perguntas acerca do comportamento que produz reforço, como ocorreu na Condição 4; e, (b) o seguimento de regras discrepantes tem maior probabilidade de deixar de ocorrer quando a regra é apresentada na forma de uma sugestão e o participante é solicitado a responder perguntas acerca do comportamento que produz reforço, como ocorreu na Condição 1.

Uma implicação desses resultados, de acordo com Albuquerque et al. (2011), é que o seguimento de regras ficaria sob o controle das propriedades formais das regras que relatam, implícita ou explicitamente, consequências futuras, denominadas de "consequências mediadas socialmente", tais como a aprovação do seguimento e a desaprovação do não seguimento de regra. Deve ser esclarecido que a descrição de consequências futuras por uma regra faz parte da regra e, dessa forma, pode exercer controle sobre a ocorrência do comportamento especificado pela regra como um estímulo verbal constituinte da regra, isto é, como estímulo antecedente verbal, mas não como uma consequência imediata do comportamento. Já o evento futuro, descrito pela regra, não pode exercer controle sobre o comportamento sob o controle antecedente da regra, porque o comportamento não pode ficar sob o controle de um evento que ainda não ocorreu (Albuquerque et al., 2011; Albuquerque \& Paracampo, 2010; Paracampo et al., 2013).

De acordo com Albuquerque et al. (2011), no estudo em análise, as regras não descreviam explicitamente as consequências futuras, mas implicitamente indicavam tais consequências. A sugestão indicava que o experimentador não iria se importar e a ordem indicava que o 
experimentador iria se importar se a regra fosse seguida ou não. Isto considerando que a sugestão especificava: "Quando eu mostrar estes objetos para você, faça o que achar melhor para você. Se você quiser, você pode fazer o seguinte: ...”. E que a ordem especificava: “Quando eu mostrar estes objetos para você, eu quero que você faça o seguinte: . .." Assim, o experimentador se importar, ou não, com a ocorrência, ou não, do seguimento de regra seria a consequência futura implicitamente descrita na regra (Albuquerque et al., 2011).

Para os autores, então, no estudo em análise, os participantes tenderam a deixar de seguir a sugestão e tenderam a seguir a ordem, em parte, porque estas regras, implicitamente em suas propriedades formais, indicavam consequências futuras diferentes para o não seguimento de regra.

O não seguir a sugestão era permitido e, portanto, não implicava em desobedecer ao experimentador. Já o não seguir a ordem não era permitido e, portanto, implicava em desobedecer ao experimentador. Assim, o não seguir a sugestão teria ocorrido, em parte, porque as propriedades formais dessa regra indicavam que o não seguimento de regra não produziria desaprovação social, isto é, indicavam que o experimentador não iria se importar caso o não seguimento de regra ocorresse. Já o seguir a ordem teria ocorrido, em parte, porque as propriedades formais dessa regra indicavam que o seguimento de regra produziria aprovação social, isto é, indicavam que o experimentador queria que o seguimento de regra ocorresse. (Albuquerque et al., 2011, p. 38)

Uma questão que surge é se os resultados encontrados por Albuquerque et al. (2011) seriam replicados, caso os participantes fossem expostos a variáveis que favorecessem o abandono do seguimento de regra discrepante das contingências, tais como as variáveis a que foram expostos os participantes da Condição 2 do Experimento 2 do estudo de Albuquerque et al. (2006) e os participantes do Grupo 1 do estudo de Oliveira e Albuquerque (2007) ${ }^{1}$. Nesses dois estudos, que usaram um procedimento de escolha de acordo com o modelo similar ao usado por Albuquerque et al. (2011), 100\% dos participantes deixaram de seguir a regra discrepante quando foram expostos a uma história em que o comportamento alternativo ao especificado pela regra discrepante das contingências era estabelecido por reforço diferencial e mantido em esquema de reforço contínuo (CRF).

Os procedimentos usados nesses três estudos, embora similares, apresentam algumas diferenças. Diferentemente do procedimento usado no estudo de Albuquerque et al. (2011), no estudo de Albuquerque et al. (2006) e no de Oliveira e Albuquerque (2007): (a) foi usado um esquema

${ }^{1}$ Para facilitar a descrição, a partir desse momento, a Condição 2 do Experimento 2 do estudo de Albuquerque et al. (2006) e o Grupo 1 do estudo de Oliveira e Albuquerque serão citadas como Albuquerque et al. (2006) e Oliveira e Albuquerque (2007), respectivamente.
CRF para reforçar o comportamento alternativo ao especificado pela regra discrepante, antes e depois da apresentação da regra; (b) não foram apresentadas perguntas aos participantes; (c) as propriedades formais das regras não foram manipuladas, uma vez que a regra discrepante só foi apresentada na forma de ordem; e, (d) a ordem apresentada especificava: "Quando eu mostrar estes objetos para você, você deve fazer o seguinte: . .", enquanto no estudo de Albuquerque et al. (2011) a ordem especificava: "Quando eu mostrar estes objetos para você, eu quero que você faça o seguinte: ...".

Assim, não ficam claros quais seriam os efeitos das manipulações de perguntas e das propriedades formais das regras (as manipulações que foram feitas no estudo de Albuquerque et al., 2011) sobre o seguimento de regras discrepantes, caso os efeitos de tais manipulações fossem investigados quando os participantes fossem expostos a uma história em que o comportamento alternativo ao especificado pela regra discrepante das contingências fosse estabelecido por reforço diferencial e mantido em CRF.

Tal investigação é importante porque ela permite testar, tanto a generalidade da proposição de Albuquerque et al. (2011), que sugere que as propriedades formais das regras, tais como as consequências sociais futuras implicitamente relatadas em regras, podem interferir na ocorrência e manutenção do seguimento de regras, quanto a proposição de Albuquerque et al. (2006) e Oliveira e Albuquerque (2007), que sugere que a história do comportamento alternativo ao especificado pela regra discrepante das contingências, bem como o esquema que reforça o não seguimento da regra, podem interferir no seguimento de regras discrepantes das contingências. Por exemplo, tal investigação permite avaliar se o seguimento da regra discrepante na forma de ordem seria mantido (como ocorreu no estudo de Albuquerque et al., 2011), ou seria abandonado (como ocorreu nos estudos de Albuquerque et al., 2006; e, Oliveira \& Albuquerque, 2007).

A investigação proposta também é importante porque ela permite avaliar as condições sob as quais perguntas, acerca do comportamento que produz reforço, podem facilitar, ou não, a discriminação da discrepância entre as consequências descritas na regra e as consequências produzidas pelo seguimento de regras.

Diante disso, o presente estudo, especificamente, teve como objetivo investigar os efeitos, sobre o seguimento de regras discrepantes das contingências, da história do comportamento alternativo ao especificado pela regra, estabelecido por reforço diferencial e mantido em CRF; das propriedades formais das regras; e, de perguntas acerca do comportamento que produz reforço. Para tanto, foi feita uma replicação sistemática do procedimento usado por Albuquerque et al. (2011). De modo que o procedimento usado no presente estudo diferiu do usado no estudo anterior (Albuquerque et al., 2011) em um único aspecto: enquanto no estudo anterior foi usado um esquema FR 4, no presente estudo foi usado um esquema CRF. Este procedimento tem a vantagem de tornar os dados do 
Gonçalves, A. S., Albuquerque, L. C. \& Paracampo, C. C. P. (2015). Controle por Regras: Efeitos de Perguntas, Propriedades Formais de Regras e Esquemas de Reforço.

presente estudo comparáveis aos dos estudos anteriores (Albuquerque et al., 2011; Albuquerque et al., 2006; Oliveira \& Albuquerque, 2007).

No presente estudo, será dito que o comportamento que se seguir à apresentação de uma regra foi estabelecido por regra, quando a combinação de duas condições for satisfeita:

1. O comportamento observado for o especificado na regra, emitido na presença dos estímulos descritos pela regra e,

2. Este comportamento ocorrer antes mesmo que as consequências programadas no experimento possam exercer algum efeito sobre ele.

Será dito que o comportamento observado foi estabelecido pelas contingências de reforço programadas no experimento quando a combinação de duas condições for satisfeita:

1. O comportamento observado for o reforçado e,

2. Este comportamento ocorrer independentemente de uma descrição antecedente verbal, especificando que comportamento na presença de que estímulo poderá ser reforçado.

E será dito que a história experimental exerceu controle quando o padrão de respostas apresentado em uma dada fase de o experimento continuar sendo apresentado na fase subsequente.

\section{Método}

\section{Participantes}

Participaram da pesquisa 24 estudantes universitários, sem história experimental prévia, de diversos cursos (exceto o de Psicologia), homens e mulheres, com idades variando entre 18 e 30 anos, matriculados em diferentes semestres. Todos foram voluntários e assinaram o Termo de Consentimento Livre e Esclarecido que, de modo geral, especificava que o objetivo da pesquisa era investigar processos de aprendizagem comuns a todas as pessoas e que caso o participante se sentisse desconfortável ou incomodado, por qualquer motivo, ele poderia interromper a sua participação na pesquisa a qualquer momento e retirar seu consentimento.

\section{Equipamento e Material}

Foi utilizada uma mesa de madeira, medindo $150 \times 78$ x $70 \mathrm{~cm}$. Fixada à mesa, de modo a dividi-la ao meio em todo o seu comprimento, havia um anteparo com espelho unidirecional de $150 \times 60 \mathrm{~cm}$, fixado em uma moldura de madeira e localizado $13 \mathrm{~cm}$ acima do tampo da mesa. No centro do anteparo, junto ao tampo da mesa, havia uma abertura retangular de $45 \times 3 \mathrm{~cm}$. Dois centímetros acima e ao centro dessa abertura havia um contador operado pelo experimentador e com os dígitos voltados para o participante. Visível ao participante estava instalada no anteparo uma lâmpada transparente de 5 watts. Uma etiqueta de papel com a frase impressa "Você ganhou um ponto" estava colocada no anteparo acima dessa lâmpada. Uma lâmpada fluorescente de 15 watts estava instalada na borda superior e ao centro do anteparo. A mesa estava situada no centro de uma sala com refrigeração.

Os estímulos modelo e de comparação eram peças de madeira (blocos lógicos da marca Funbec), variando em três dimensões: forma (quadrado, círculo, retângulo e triângulo), cor (azul, vermelha e amarela) e espessura (grossa e fina). Essas peças de madeira formavam 40 diferentes arranjos de estímulos, cada arranjo era constituído de um estímulo modelo e três estímulos de comparação. Cada estímulo de comparação apresentava apenas uma dimensão - cor (C), espessura (E), ou forma (F) - em comum com o estímulo modelo e diferia nas demais. Os arranjos de estímulos, previamente preparados, ficavam sobre a mesa, ao lado do experimentador, na ordem em que seriam apresentados em cada tentativa. Os estímulos eram apresentados ao participante através da abertura na base do anteparo divisor da mesa, em uma bandeja de madeira em forma de ' $T$ '. Na parte final do cabo dessa bandeja, quatro ripas de madeira formavam um quadrado no qual era colocado o estímulo modelo. Na base retangular, dividida por ripas de madeira em três quadrados, eram apresentados os três estímulos de comparação. As respostas de apontar para os estímulos de comparação, bem como eventuais verbalizações emitidas pelos participantes eram registradas pelo experimentador em um protocolo previamente preparado e eram também gravadas por uma filmadora, para análises posteriores. Os reforçadores utilizados eram pontos, registrados no contador. Cada ponto valia $\mathrm{R} \$ 0,05$ (cinco centavos de real).

As instruções foram gravadas em um computador e transferidas para um gravador de áudio. As instruções eram apresentadas ao participante por intermédio de fones de ouvido, conectados ao gravador de áudio.

\section{Procedimento}

Durante as sessões experimentais, participante e experimentador ficavam sentados à mesa de frente um para o outro, separados pelo anteparo divisor da mesa. A lâmpada na borda superior do anteparo ficava constantemente acesa, voltada para o participante, de maneira a assegurar que seu lado apresentasse iluminação em maior intensidade, garantindo que apenas as ações emitidas pelo participante, bem como o arranjo dos estímulos apresentados, pudessem ser observadas através do espelho. O experimentador, em algumas sessões, inicialmente apresentava ao participante uma determinada instrução e em seguida apresentava os arranjos de estímulos, em outras, apresentava apenas os arranjos de estímulos. As sessões duravam em média 30 min. O intervalo entre sessões era de aproximadamente 5 min. Foram realizadas três sessões, em um mesmo dia, com cada participante.

Em cada tentativa, o experimentador apresentava um dos 40 arranjos de estímulos. Na presença desses estímulos, o participante deveria apontar para cada um dos três estímulos de comparação em uma dada sequência. Caso a sequência de respostas emitida estivesse de acordo 
com as contingências de reforço programadas (sequência correta), um ponto era acrescentado no contador, a lâmpada transparente era acesa e apagada, e a bandeja com o arranjo de estímulos era retirada. Caso a sequência de respostas fosse incorreta, a lâmpada transparente não era acesa e a bandeja com o arranjo de estímulos era retirada, sem que fosse acrescentado um ponto no contador. Havia um intervalo variável de aproximadamente $5 \mathrm{~s}$ entre uma tentativa e outra. Os pontos eram registrados cumulativamente, no contador, apenas dentro de uma mesma sessão. No entanto, a partir da segunda sessão, logo após entrar na sala experimental, o participante era informado pelo experimentador sobre o número total de pontos obtidos nas sessões anteriores.

\section{Orientações Preliminares}

Na primeira sessão, quando participante e experimentador entravam na sala, a bandeja com um arranjo de estímulos estava sobre a mesa, portanto, visível ao participante. O experimentador pedia ao participante que se sentasse na cadeira e que colocasse os fones de ouvido. No outro lado da mesa, o auxiliar de pesquisa ligava o gravador. Por meio dos fones, o participante passava a ouvir as seguintes orientações:

Este objeto, aqui em cima, é um modelo. Estes três objetos, aqui em baixo, são para você comparar com o modelo. Nós vamos chamar estes três objetos, aqui em baixo, de objetos de comparação. Observe que cada um destes três objetos de comparação tem uma única propriedade comum ao modelo. Veja. Este só tem a espessura comum ao modelo. Este aqui só tem a cor comum ao modelo. E este aqui só tem a forma igual ao modelo. Durante a pesquisa você poderá ganhar pontos que serão trocados por dinheiro. Quando você ganhar pontos, os pontos sempre aparecerão aqui neste contador. Veja como os pontos aparecem no contador [o auxiliar, que se encontrava no outro lado da mesa, acionava o contador e acendia a lâmpada cinco vezes]. Quando você não ganhar pontos, nenhum ponto será acrescentado no contador. Entendeu?

O experimentador, ao lado do participante, sempre apontava com o dedo para cada um dos estímulos a que a gravação se referia. Esse procedimento ocorria apenas no início da primeira sessão.

\section{Regras}

A seguir, o experimentador se deslocava em direção à sua cadeira. Separado do participante pelo anteparo com espelho unidirecional, o experimentador também colocava os seus fones de ouvido e, dependendo da sessão experimental, entregava ao participante, pela abertura na base do anteparo, uma folha de papel contendo uma das seguintes instruções (regras) impressas:

Instrução Mínima. Instrução que não especificava sequência de respostas.

"Aponte com o dedo em sequência para cada um dos três objetos de comparação".
Regra Discrepante (apresentada na forma de Sugestão). Regra cujo comportamento de segui-la não produzia ponto.

Quando eu mostrar estes objetos para você, faça o que achar melhor para você. Se você quiser, você pode fazer o seguinte: Primeiro aponte para a mesma forma, depois para a mesma cor e em seguida para a mesma espessura. Fazendo isso, você poderá ganhar pontos que serão mostrados no contador à sua frente. Cada ponto que você ganhar será trocado por R $\$ 0,05$ (cinco centavos de real), mas apenas no final da pesquisa.

Regra Discrepante (apresentada na forma de Ordem). Regra cujo comportamento de segui-la não produzia ponto.

Quando eu mostrar estes objetos para você, eu quero que você faça o seguinte: Você deve apontar primeiro para a mesma espessura, depois para a mesma cor e em seguida para a mesma forma. Fazendo isso, você poderá ganhar pontos que serão mostrados no contador à sua frente. Cada ponto que você ganhar será trocado por $\mathrm{R} \$ 0,05$ (cinco centavos de real), mas apenas no final da pesquisa.

Imediatamente após entregar ao participante a folha de papel contendo uma instrução impressa, o experimentador ligava o gravador de áudio e, por intermédio dos fones de ouvido, o participante passava a ouvir uma fita, previamente gravada, com a voz do experimentador lendo a instrução. Na gravação, o experimentador solicitava ao participante, ora que acompanhasse a sua leitura, ora que ele lesse sozinho, silenciosamente. Desse modo, o participante lia a instrução por três vezes seguidas no início das Fases 1, 2 e 3. Após a última leitura, a gravação solicitava ao participante que devolvesse a folha com a instrução. Logo após receber a folha com a instrução, o experimentador removia a bandeja, voltava a apresentar a bandeja com um novo arranjo de estímulos, e dizia: "Comece a apontar".

\section{Perguntas}

Imediatamente após a apresentação da instrução mínima, em algumas condições (descritas a seguir), o experimentador entregava ao participante, pela abertura na base do anteparo, uma folha de papel contendo o seguinte: "Qual a sequência correta?".

"Quantas vezes você têm que apontar, consecutivamente, na sequência correta para ganhar um ponto?". "Quando você descobrir as respostas para estas duas perguntas, escreva as suas respostas nesta mesma folha de papel. Imediatamente após você escrever as respostas, devolva-me esta folha pela mesma abertura que você a recebeu".

\section{Delineamento Experimental}

Os participantes foram distribuídos em quatro condições experimentais, conforme a Tabela 1. Cada condição, realizada com seis participantes, era constituída de três fases. Nas quatro condições, a Fase 1 era iniciada com a 
Gonçalves, A. S., Albuquerque, L. C. \& Paracampo, C. C. P. (2015). Controle por Regras: Efeitos de Perguntas, Propriedades Formais de Regras e Esquemas de Reforço.

apresentação da instrução mínima e as Fases 2 e 3 eram iniciadas com a apresentação de uma das duas regras discrepantes das contingências (sugestão ou ordem). A Fase 1 era encerrada imediatamente após a ocorrência de 160 tentativas, desde que o participante tivesse emitido no mínimo 10 sequências corretas consecutivamente. Caso o participante não atingisse esse critério de desempenho de emitir 10 sequências corretas consecutivamente, a fase era encerrada após as 160 tentativas e o participante era excluído da pesquisa. Cada uma das Fases 2 e 3 era encerrada após ser completada uma sessão, que consistia de 80 tentativas. O início e o encerramento de uma sessão eram marcados, respectivamente, pela entrada e saída do participante da sala experimental. Durante cada uma das três fases, a única sequência de respostas reforçada (correta) era a sequência cor (C), espessura (E) forma (F). Esta sequência era reforçada com pontos trocáveis por dinheiro. A emissão de qualquer outra sequência não era reforçada. A sequência CEF era reforçada em esquema de reforço contínuo (CRF).

Tabela 1

Esquema do Procedimento

\begin{tabular}{lcccccc}
\hline & & Condição 1 & & \multicolumn{3}{c}{ Condição 2 } \\
\hline & Fase 1 & Fase 2 & Fase 3 & Fase 1 & Fase 2 & Fase 3 \\
\hline Perguntas & Sim & Não & Não & Não & Não & Não \\
Sequência instruída & Nenhuma & FCE & ECF & Nenhuma & FCE & ECF \\
Sequência reforçada em CRF & CEF & CEF & CEF & CEF & CEF & CEF \\
Forma da regra & Não & Sugestão & Ordem & Não & Sugestão & Ordem \\
\hline & & Condição 3 & & & Condição 2 & \\
\hline & Fase 1 & Fase 2 & Fase 3 & Fase 1 & Fase 2 & Fase 3 \\
\hline Perguntas & Sim & Não & Não & Não & Não & Não \\
Sequência instruída & Nenhuma & ECF & FCE & Nenhuma & ECF & FCE \\
Sequência reforçada em CRF & CEF & CEF & CEF & CEF & CEF & CEF \\
Forma da regra & Não & Ordem & Sugestão & Não & Ordem & Sugestão \\
\hline
\end{tabular}

Nota . Cada condição foi realizada com seis participantes. $\mathrm{C}=$ resposta à dimensão cor. $\mathrm{E}=$ resposta à espessura. $\mathrm{F}=$ resposta à forma. $\mathrm{CRF}=$ esquema de reforço contínuo.

As quatro condições diferiam em dois aspectos: (a) quanto à apresentação, ou não, das perguntas na Fase 1; e, (b) quanto à ordem de apresentação das regras discrepantes (sugestão ou ordem) nas Fases 2 e 3.

Nas Condições 1 (com perguntas/sugestão/ordem) e 3 (com perguntas/ordem /sugestão), imediatamente após apresentar a instrução mínima, no início da Fase 1, o experimentador entregava ao participante a folha de papel contendo as perguntas e, em seguida, passava a apresentar os arranjos de estímulos. Caso o participante devolvesse a folha de papel apresentando respostas incorretas às perguntas, o experimentador lhe entregava uma nova folha, contendo as mesmas perguntas. Esse procedimento era repetido no máximo 10 vezes. Se o participante não respondesse corretamente às perguntas nessas 10 oportunidades, sua participação no experimento era encerrada na Fase 1. Assim, nas Condições 1 e 3, só eram expostos à Fase 2 os participantes que tivessem atingido esse critério e o critério de desempenho para o encerramento da Fase 1 supracitado.
Nas Condições 2 (sem perguntas/sugestão/ordem) e 4 (sem perguntas/ordem/sugestão), o experimentador não entregava ao participante a folha de papel contendo as perguntas.

Nas Condições 1 (com perguntas/sugestão/ordem) e 2 (sem perguntas/sugestão/ordem), a Fase 2 era iniciada com a apresentação da regra discrepante na forma de sugestão e a Fase 3 era iniciada com a apresentação da regra discrepante na forma de ordem. Nas Condições 3 (com perguntas/ordem /sugestão) e 4 (sem perguntas/ordem / sugestão), a Fase 2 era iniciada com a apresentação da regra discrepante na forma de ordem e a Fase 3 era iniciada com a apresentação da regra discrepante na forma de sugestão.

\section{Comparação dos Registros e Término da Participação do Estudante no Experimento \\ Nas quatro condições, após a terceira sessão, um observador independente comparava o registro feito pelo experimentador com o registro feito pela filmadora. Caso houvesse $100 \%$ de concordância entre os registros, os}


dados eram considerados para análise. Caso contrário, os dados eram descartados. A participação do estudante no experimento era encerrada depois de ser atingido o crité- rio de encerramento da Fase 3, ou caso o estudante não atingisse os critérios estabelecidos na Fase 1, relativos ao encerramento de fase e às respostas às perguntas.

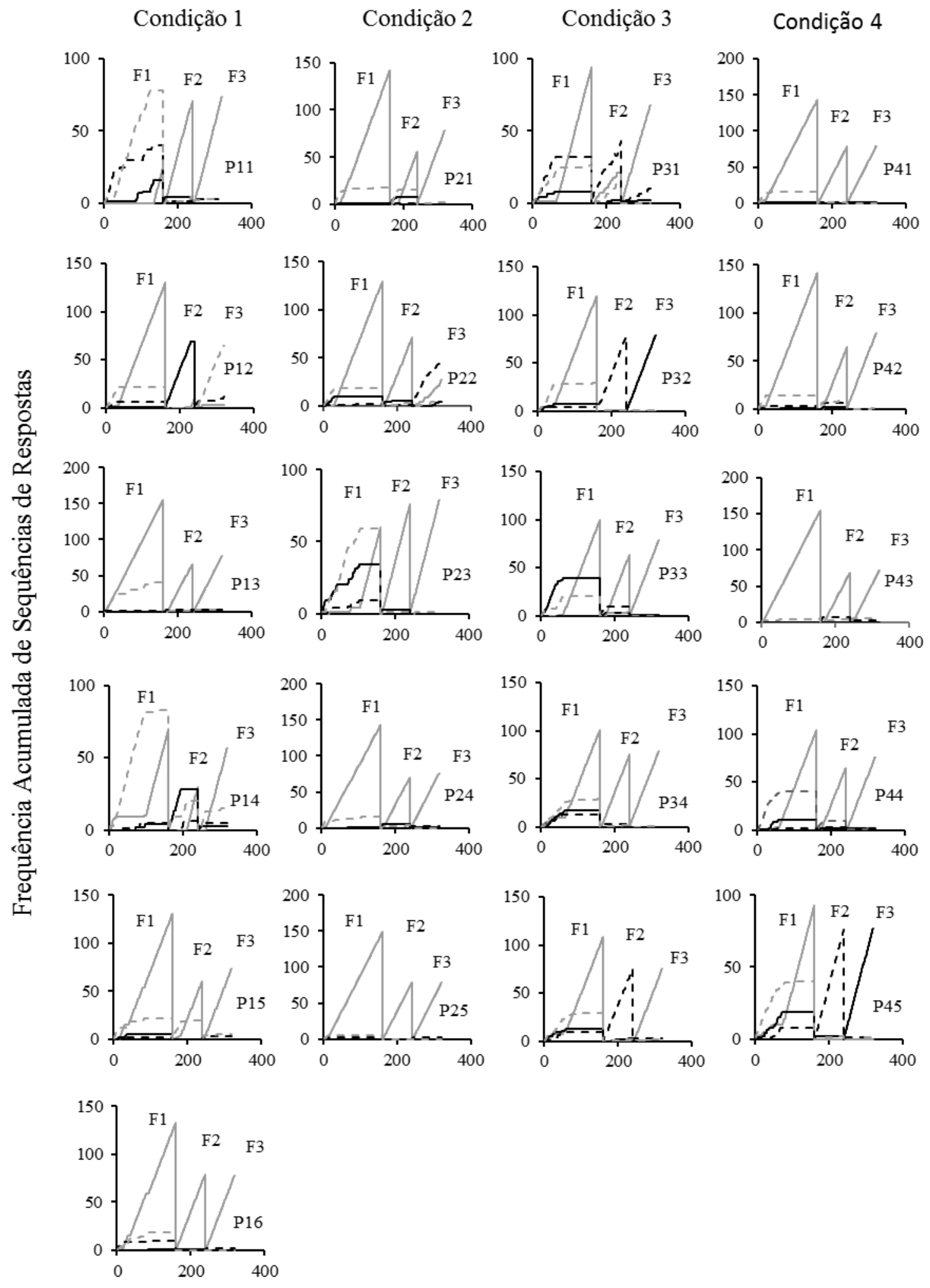

Tentativas

Figura 1. Frequência acumulada de sequências emitidas pelos participantes (P) nas Fases (F) 1, 2 e 3 de cada condição. 
Gonçalves, A. S., Albuquerque, L. C. \& Paracampo, C. C. P. (2015). Controle por Regras: Efeitos de Perguntas, Propriedades Formais de Regras e Esquemas de Reforço.

\section{Resultados}

A Figura 1 apresenta os dados das Condições 1, 2, 3 e 4. A figura mostra a frequência acumulada de sequências de respostas emitidas pelos participantes $(\mathrm{P})$, durante as Fases (F) 1, 2 e 3 em cada condição. Quebra na curva acumulada indica mudança de fase. A linha cinza sólida indica a sequência (CEF) correta (reforçada), ou seja, o comportamento alternativo ao especificado pela regra. A linha cinza tracejada indica outras sequências, que não as sequências CEF, ECF e FCE. A linha preta tracejada indica a sequência $\mathrm{ECF}$, especificada pela regra discrepante, em forma de ordem, na Fase 3 das Condições 1 e 2 e na Fase 2 das Condições 3 e 4 . A linha preta sólida indica a sequência FCE, especificada pela regra discrepante, em forma de sugestão, na Fase 2 das Condições 1 e 2 e na Fase 3 das Condições 3 e 4.

De modo geral, independentemente da condição experimental, 21 dos 24 participantes, atingiram o critério de desempenho para o encerramento da Fase 1 e foram expostos às Fases 2 e 3 . Na Fase 2, todos os 21 participantes iniciaram a fase seguindo a regra discrepante.

$\mathrm{Na}$ Condição 1 (com perguntas/sugestão/ordem), Fase 2, pode-se observar que todos os seis participantes (P11, P12, P13, P14, P15 e P16) abandonaram o seguimento da sugestão e passaram a emitir o comportamento alternativo ao especificado pela regra (a sequência $\mathrm{CEF}$ [correta], estabelecida na Fase 1). Na Fase 3, todos os seis participantes deixaram de seguir a ordem e cinco (P11, $\mathrm{P} 13, \mathrm{P} 14, \mathrm{P} 15$ e P16) desses seis passaram a responder na sequência correta.

$\mathrm{Na}$ Condição 2 (sem perguntas/sugestão/ordem), Fase 2 , pode-se observar que todos os cinco participantes (P21, P22, P23, P24 e P25) abandonaram o seguimento da sugestão e passaram a emitir a sequência correta. Na Fase 3, quatro participantes (P21, P23, P24 e P25) passaram a responder na sequência correta. Já o Participante P22 passou a Fase 3 variando, ora seguindo a ordem, ora emitindo a sequência correta (reforçada com ponto).

$\mathrm{Na}$ Condição 3 (com perguntas/ordem/sugestão), houve variabilidade na Fase 2. Dois participantes (P33 e P34) deixaram de seguir a ordem e passaram a emitir a sequência reforçada. Dois participantes (P32 e P35) seguiram a ordem praticamente durante toda a fase. E um participante (P31) também seguiu a ordem durante a maior parte das tentativas da Fase 2, mas às vezes ele emitia, ora a sequência correta, ora outras sequências. Na Fase 3, P31, P33, P34 e 35 terminaram a fase emitindo a sequência correta e, portanto, não seguindo a sugestão. Já P32, continuou seguindo a regra discrepante, agora em forma de sugestão.

$\mathrm{Na}$ Condição 4 (sem perguntas/ordem/sugestão), na Fase 2, pode-se observar que quatro participantes ( $\mathrm{P} 41$, $\mathrm{P} 42$, P43 e P44) deixaram de seguir a ordem e passaram a emitir a sequência correta. $\mathrm{P} 45$ foi o único que continuou seguindo a ordem. Na Fase 3, P41, P42, P43 e P44 responderam corretamente e, portanto, não seguiram a sugestão. P45 continuou seguindo regra, no caso, a sugestão.

\section{Discussão}

O presente estudo procurou avaliar os efeitos, sobre o seguimento de regras discrepantes das contingências de reforço, da história do comportamento alternativo ao especificado pela regra; das propriedades formais das regras; e, de perguntas acerca do comportamento que produz reforço.

Em relação aos efeitos da história do comportamento alternativo ao especificado pela regra, pode-se dizer que as diferenças de resultados do presente estudo e do estudo anterior (Albuquerque et al., 2011), podem ser atribuídas, em parte, às diferenças entre os procedimentos usados nesses estudos, isto é, às diferenças entre as histórias do comportamento alternativo ao especificado pela regra e entre os esquemas programados para reforçar o não seguimento da regra (ou seja, o comportamento alternativo ao especificado pela regra). Assim, é possível que no presente estudo um percentual maior de participantes tenha abandonado o seguimento de regra (100\% abandonaram o seguimento da sugestão e $60 \%$ abandonaram o seguimento da ordem) do que no estudo anterior ( $83 \%$ abandonaram o seguimento da sugestão e $20 \%$ abandonaram o seguimento da ordem), porque: (a) na história do presente estudo, o comportamento alternativo ao especificado pela regra era reforçado em CRF, enquanto na história do estudo anterior esse comportamento alternativo era reforçado em FR 4; e (b) o esquema que reforçava o não seguimento da regra era CRF, no presente estudo, e FR 4, no estudo anterior. Essa análise sugere que os efeitos de regras dependem, em parte, da história do comportamento alternativo ao especificado pela regra e do esquema programado para reforçar esse comportamento alternativo.

Um exemplo prático pode ajudar a esclarecer como essa história do comportamento alternativo ao especificado pela regra exerce a sua função de impedir o controle subsequente por regras. Suponha um ouvinte que acaba de ser exposto a seguinte regra: "Você tem diabetes e de agora em diante deve passar a tomar remédio, fazer dieta e exercícios físicos regularmente". Neste caso, a história do comportamento alternativo ao especificado por essa regra, seria a história de vida sedentária e de comer alimentos pouco saudáveis. Essa história, tal como a história do presente estudo, poderia contribuir para impedir a ocorrência do seguimento subsequente das regras (Albuquerque et al., 2014; Najjar, Albuquerque, Ferreira, \& Paracampo, 2014).

Quanto aos efeitos das propriedades formais das regras, pode-se dizer que o seguimento da regra na forma de ordem foi mais provável de ser mantido do que o seguimento da rega na forma de sugestão, tanto no presente estudo quanto no estudo de Albuquerque et al. (2011), devido, em grande parte, às diferenças entre as propriedades formais das regras. Em outras palavras, a ordem foi mais provável de ser seguida do que a sugestão nesses dois estudos, em grande parte, porque as propriedades formais da ordem indicavam que o experimentador fazia questão que essa regra (a ordem) fosse seguida, enquanto que as propriedades formais da sugestão indicavam que o experimentador não fazia 
questão que essa regra (a sugestão) fosse seguida ou não.

Uma evidência adicional do controle pelas propriedades formais das regras pode ser encontrada quando os dados do presente estudo são comparados com os dados dos estudos de Albuquerque et al. (2006) e Oliveira e Albuquerque (2007). Nesses dois estudos, 100\% dos participantes deixaram de seguir a regra discrepante quando foram expostos a uma história em que o comportamento alternativo ao especificado por essa regra era estabelecido por reforço diferencial e mantido em CRF, antes de a apresentação da regra, e o não seguimento de regra era reforçado em CRF. No presente estudo, quando foram expostos a tais condições, apenas $60 \%$ dos participantes abandonaram o seguimento da ordem. Tais diferenças de resultados podem ser atribuídas às diferenças entre as propriedades formais das regras discrepantes em forma de ordem utilizadas nestes três estudos. Nos estudos anteriores (Albuquerque et al., 2006; e, Oliveira \& Albuquerque, 2007), a ordem especificava: "Quando eu mostrar estes objetos para você, você deve fazer o seguinte: . ..”, enquanto no presente estudo a ordem especificava: "Quando eu mostrar estes objetos para você, eu quero que você faça o seguinte: . . .". No presente estudo, portanto, pode-se supor que a ordem deixava mais explícito, do que nesses dois estudos anteriores, que o experimentador queria (fazia questão, se importava) que a regra fosse seguida e esse fato pode ter contribuído para que $40 \%$ dos participantes tivessem seguido a regra, apesar das condições favoráveis para o não seguimento de regra. Esses resultados sugerem que os efeitos de regras podem competir com os efeitos da história do comportamento alternativo ao especificado pela regra e, como resultado dessa competição, algumas vezes essa história pode impedir o controle por regras (caso dos participantes que deixaram de seguir a regra) e outras vezes o inverso pode ocorrer (caso dos participantes que seguiram a regra).

Essa análise dos resultados têm implicações para o esclarecimento do papel das consequências na explicação do comportamento. Por exemplo, na análise do comportamento, a noção sobre por que as pessoas se comportam da maneira como se comportam, é em grande parte orientada pelas proposições de Skinner, que considera que todo comportamento é determinado, direta ou indiretamente, pelas consequências (Skinner, 1974). Por essa proposição, uma regra ("Faça exercícios físicos", por exemplo) poderia evocar o comportamento por ela especificado, mas não alteraria a probabilidade de esse comportamento vir a ocorrer no futuro. Seriam as consequências que alterariam a probabilidade de o comportamento de seguir regra ocorrer no futuro. Ou seja, o que determinaria se um ouvinte continuaria, ou não, seguindo a regra "Faça exercícios físicos", por exemplo, não seria a regra, mas a história de exposição às consequências para o comportamento de seguir regras (Skinner, 1969). Os estudiosos da área concordam com essa proposição (Baum, 1994/1999; Baumann, Abreu-Rodrigues, \& Souza, 2009; Baron \& Galizio, 1983; Catania et al., 1989; Cerutti, 1989; Chase \&
Danforth, 1991; Hayes et al., 1986; Martinez \& Tomayo, 2005; Newman et al., 1995; Okoughi, 1999; Perez, Reis, \& de Souza, 2009; Torgrud \& Holborn, 1990; Wulfert et al., 1994).

No entanto, essa proposição de Skinner (1969) não deixa claro se as consequências que alterariam a probabilidade de o comportamento de seguir regra ocorrer no futuro seriam as consequências imediatas contatadas pelo comportamento de seguir e de não seguir regra ou as consequências futuras relatadas em regras. É importante fazer a distinção entre essas duas classes de consequências porque tal distinção implica no estabelecimento de limites entre o que é controle por regras e o que é controle por contingências. Assim, pode-se dizer que uma consequência futura relatada em uma regra pode exercer controle sobre o comportamento porque ela está presente no momento em que a regra é apresentada e, dessa maneira, como um estímulo antecedente verbal participante da regra, essa consequência pode ser contatada pelo comportamento. Já o evento futuro relatado, em si mesmo, não exerce controle porque este evento não é contatado pelo comportamento no momento em que a regra é apresentada. Quando o evento relatado é contatado pelo comportamento, ele não é contatado enquanto evento futuro, mas sim como uma consequência imediata do comportamento e é dessa forma que ele pode exercer controle. Desse modo, a regra funcionaria como substituto atual do evento futuro por ela relatado (Albuquerque et al., 2014).

Para distinguir os efeitos de estímulos que constituem a regra (relato de consequências futuras, por exemplo) dos efeitos de outros estímulos (consequências imediatas do comportamento, por exemplo) sobre o comportamento especificado pela regra, Albuquerque e colaboradores (Albuquerque, 2005; Albuquerque et al., 2011; Albuquerque \& Paracampo, 2010; Albuquerque et al., 2013; Albuquerque et al., 2014; Paracampo et al., 2013) sugeriram o uso do termo justificativas ${ }^{2}$. Esse termo descreve os efeitos dos estímulos que constituem a regra, isto é, as propriedades formais da regra. Considerando isto, pode-se sugerir que, no presente estudo e no estudo de Albuquerque et al. 
Gonçalves, A. S., Albuquerque, L. C. \& Paracampo, C. C. P. (2015). Controle por Regras: Efeitos de Perguntas, Propriedades Formais de Regras e Esquemas de Reforço.

(2011), as justificativas diferenciais (Tipos 1, 2 e 4) para seguir (implicadas na ordem) e para seguir ou não seguir regra (implicadas na sugestão) contribuíram para alterar a probabilidade de emissão do comportamento de seguir (no caso dos $40 \%$ dos participantes que seguiram a ordem) e de não seguir regra (no caso da sugestão).

Esses resultados apoiam a proposição que sugere que regras podem alterar a probabilidade de o seguimento de regras vir a ocorrer no futuro (Albuquerque et al., 2011; Albuquerque \& Paracampo, 2010; Albuquerque et al., 2013; Albuquerque et al., 2014; Paracampo et al., 2013). Por essa proposição ${ }^{3}$, quando um falante apresenta uma regra para um ouvinte, em geral, ele também apresenta justificativas para a ocorrência e/ou manutenção do seguimento da regra previamente apresentada. E tais justificativas podem alterar a probabilidade de o seguimento da regra vir a ocorrer no futuro. Por exemplo, um falante pode dizer a um ouvinte, com planos de fazer a sua primeira viagem: "Não vá para $\mathrm{X}$ porque lá não tem praia. Vá para $\mathrm{Y}$ e lá você terá tudo o que deseja". E o ouvinte segue a recomendação do falante. Neste exemplo, as justificativas (do Tipo 1) alterariam a probabilidade de o ouvinte ir para $\mathrm{Y}$.

Considerando isso e os resultados do presente estudo, pode-se sugerir que: (a) a consequência futura relatada, implícita ou explicitamente, em uma regra pode exercer controle como regra, isto é, como um estímulo antecedente verbal (Albuquerque et al., 2014; Paracampo et al., 2013) e, portanto, não deveria ser considerada como uma "Contingência verbal" (Skinner, 1969) ou "Contingência última" (Baum, 1994/1999). (b) Um comportamento (viajar, por exemplo) e uma consequência futura (ter as coisas que deseja, por exemplo) podem fazer parte de uma contingência. Mas quando um falante descreve essa relação para um ouvinte e o comportamento especificado pela regra ocorre, antes de entrar em contato com o evento descrito, tal comportamento deve ser considerado como controlado por regra. Quando o comportamento especificado pela regra entra em contato com o evento futuro descrito (contato com as coisas que o ouvinte deseja), ele passa a ser controlado pela interação entre a regra e a consequência imediata contatada. Essa história pode alterar a probabilidade de esse comportamento vir a ocorrer no futuro. (c) Nem todos os eventos futuros relatados por regras são claramente passíveis de serem contatados. Por exemplo, uma pessoa pode emitir comportamentos (tal como colidir um veículo contra uma parede de concreto)

${ }^{3}$ De acordo com essa proposição, quando o comportamento é controlado por regras, são as regras que determinam a topografia do comportamento, a sua probabilidade de ocorrer no futuro e, alteram as funções dos estímulos. Já quando o comportamento é controlado por contingências, são as consequências imediatas do comportamento que exercem essas funções. Mas, funcionalmente, regras diferem das consequências imediatas, porque regras também podem evocar comportamento e as consequências imediatas não exercem esta função. Deste modo, regras também não deveriam ser chamadas de contingências verbais (Albuquerque \& Paracampo, 2010; Albuquerque et al., 2013; Albuquerque et al., 2006). sob o controle de regras relatam que, dessa forma, terá acesso ao paraíso após a morte. Assim, o seguimento de regras não seria determinado apenas pela história de exposição a consequências imediatas diferenciais para seguir e para não seguir regras (Baum, 1994/1999; Baron \& Galizio, 1983; Catania et al., 1989; Cerutti, 1989; Chase \& Danforth, 1991; Okoughi, 1999; Paracampo et al., 2014; Wulfert et al., 1994). O seguimento de regras também seria determinado pela história de exposição a justificativas diferenciais para seguir e para não seguir regras (Albuquerque et al., 2013). A diferença entre essas duas histórias é que, no primeiro caso, o seguir regras mantém contato com as suas consequências imediatas. Já no segundo caso, mantém contato com as justificativas relatadas para o seguimento da regra. A função dessas histórias pré-experimentais de seguimento de regras seria contribuir para a ocorrência do comportamento generalizado de seguir regras (Albuquerque et al., 2011). Mas isso não implica que as pessoas seguiriam qualquer regra, indiscriminadamente, apenas porque têm uma história pré-experimental de seguimento de regras. Os resultados do presente estudo mostram que o seguimento de uma ou outra regra também depende de justificativas atuais para seguir ("eu quero que você faça o seguinte: ...") e para seguir ou não seguir regras ("faça o que achar melhor para você. Se você quiser, você pode fazer o seguinte: ...") e de outras variáveis situacionais, como as consequências imediatas produzidas pelo seguir (não obtenção de pontos) e pelo não seguir regras (obtenção de pontos); e, pela história recente do comportamento alternativo ao especificado pela regra.

Essa análise deveria passar a ser considerada em estudos que frequentemente usam termos relacionados a consequências futuras, tais como, por exemplo, os estudos sobre autocontrole (Hanna \& Todorov, 2002; Hoerger \& Mace, 2006); metacontingências (Glenn, 1986; Martone \& Todorov, 2007); comportamento do consumidor (Foxall, 1998; Oliveira-Castro, 2003); e comportamento de escolha (La Piedad, Field, \& Rachlin, 2006). Também deveria ser aplicada em estudos de casos em que são usados termos (tais como "Objetivos"; "Metas"; "Compromissos"; "Intenções"; "Propósitos"; "Expectativas"; "Destino"; "Desejos", entre outros) para descrever o comportamento que, sob o controle de privações, aparenta estar sob o controle de consequências futuras, mas que está sob o controle antecedente de regras que relatam tais consequências.

Passando agora a analisar os efeitos de perguntas acerca das contingências, os resultados do estudo de Albuquerque et al. (2011) mostraram que, quando foram solicitados a responder perguntas acerca das contingências, 7 dos 11 participantes terminaram a Fase 2 não seguindo a regra discrepante. Inversamente, quando não foram solicitados a responder perguntas acerca das contingências, 8 dos 11 participantes seguiram a regra discrepante durante a maior parte da Fase 2. No presente estudo, quando foram solicitados a responder perguntas acerca das contingências, 8 dos 11 participantes terminaram a Fase 2 não seguindo a regra discrepante. Do mesmo modo, quando não foram 
solicitados a responder perguntas acerca das contingências, 9 dos 10 participantes também terminaram a Fase 2 não seguindo a regra discrepante. Esses dados, juntos, sugerem que fazer perguntas, acerca das contingências de reforço às quais as pessoas são expostas, tem maior probabilidade de facilitar a discriminação da discrepância entre as consequências relatadas na regra e as consequências imediatas produzidas pelo seguimento de regra e, dessa forma, facilitar o abandono do seguimento de regra discrepante, quando as perguntas abordam contingências difíceis de serem discriminadas (como o esquema FR 4 usado no estudo anterior) do quando as perguntas abordam contingências fáceis de serem discriminadas (como o esquema CRF usado no presente estudo).

Os dados dos participantes que responderam corretamente às perguntas também sugerem que esses participantes sabiam o que fazer na Fase 2 (seja porque foram expostos a uma regra que dizia o que eles deveriam fazer [a regra apresentada na Fase 2], seja porque aprenderam o que fazer ao longo de sua exposição às contingências programadas na Fase 1 [a história de estabelecimento do comportamento alternativo]). Na Fase 2, alguns participantes fizeram o que aprenderam por contingências (na Fase 1) e outros fizeram o que aprenderam por regra (na Fase 2). Em todos os casos, como já analisado, o que os participantes fizeram foi determinado pelas justificativas, consequências imediatas do comportamento e história do comportamento alternativo ao especificado pela regra.

Por fim, pesquisas futuras deveriam começar a testar as proposições apresentadas no presente estudo. Para tanto, recomenda-se o uso do procedimento de emparelhamento com o modelo usado no presente estudo, porque, em relação ao esquema múltiplo tradicionalmente usado nessa área (Baron \& Galizio, 1983; Hayes et al., 1986), ele tem a vantagem de oferecer várias possibilidades de combinações e recombinações entre os estímulos que constituem a regra e os estímulos que constituem as condições as quais o seguimento de regras ocorre. Essa característica do procedimento usado aqui é que permite avaliar, a cada tentativa, em um mesmo participante, os efeitos do controle por contingências de reforço; regras; e, história experimental sobre comportamentos previamente especificados.

\section{Referências}

Albuquerque, L. C. (2001). Definições de regras. In H. J. Guilhardi, M. B. B. P. Madi, P. P. Queiroz, \& M. C. Scoz (Eds.), Sobre comportamento e cognição: Expondo a variabilidade (pp. 132-140). Santo André, SP: Arbytes.

Albuquerque, L. C. (2005). Regras como instrumento de análise do comportamento. In L. C. Albuquerque (Ed.), Estudos do comportamento (pp. 143-176). Belém, PA: Editora da Universidade Federal do Pará.

Albuquerque, L. C., de Souza, D. G., Matos, M. A., \& Paracampo, C. C. P. (2003). Análise dos efeitos de histórias experimentais sobre o seguimento subsequente de regras. Acta Comportamentalia, 11, 87-126.
Albuquerque, L. C., Mescouto, W. A., \& Paracampo, C. C. P. (2011). Controle por regras: Efeitos de perguntas, sugestões e ordens. Acta Comportamentalia, 19, 19-42.

Albuquerque, L. C., \& Paracampo, C. C. P. (2010). Análise do controle por regras. Psicologia USP, 21, 253-273. doi:10.1590/S0103-65642010000200004

Albuquerque, L. C., Paracampo, C. C. P., Matsuo, G. L., \& Mescouto, W. A. (2013). Variáveis combinadas, comportamento governado por regras e comportamento modelado por contingência. Acta Comportamentalia, 21(3), 285-304.

Albuquerque, L. C., Reis, A. A., \& Paracampo, C. C. P. (2006). Efeitos de uma história de reforço contínuo sobre o seguimento de regra. Acta Comportamentalia, 14, 47-75.

Albuquerque, L. C., Silva, L. de S., \& Paracampo, C. C. P. (2014). Análise de variáveis que podem interferir no comportamento de seguir regras discrepantes. Acta Comportamentalia, 22, 51-71.

Baum, W. M. (1999). Compreender o behaviorismo: Ciência, comportamento e cultura. Porto Alegre, RS: Artmed. (Original publicado em 1994)

Baumann, A. A., Abreu-Rodrigues, J., \& Souza, A. S. (2009). Rules and self-rules: Effects of variation upon behavioral sensitivity to change. The Psychological Record, 34, 641-670. doi: $10.5167 /$ uzh-40461

Baron, A., \& Galizio, M. (1983). Instructional control of human operant behavior. The Psychological Record, 33, 495-520.

Braga, M. V. N., Albuquerque, L. C., Paracampo, C. C. P., \& Santos, J. V. (2010). Efeitos de manipulações de propriedades formais de estímulos verbais sobre o comportamento. Psicologia: Teoria e Pesquisa, 4, 129-142. doi:10.1590/ S0102-37722010000400010

Catania, A. C., Shimoff, E., \& Matthews, A. (1989). An experimental analysis of rule-governed behavior. In S. C. Hayes (Ed.), Rule-governed behavior: Cognition, contingencies, and instructional control (pp. 119-150). New York: Plenum. doi:10.1007/978-1-4757-0447-1_4

Cerutti, D. T. (1989). Discrimination theory of rule-governed behavior. Journal of the Experimental Analysis of Behavior, 51, 259-276. doi:10.1901/jeab.1989.51-259

Chase, P. N., \& Danforth, J. S. (1991). The role of rules in concept learning. In L. J. Hayes \& P. N. Chase (Eds.), Dialogues on verbal behavior (pp. 205-225). Hillsdale, NJ: Lawrence Erlbaum.

Foxall, G. R. (1998). Radical behaviorist interpretation: Generating and evaluating an account of consumer behavior. The Behavior Analyst, 21, 321-354.

Galizio, M. (1979). Contingency-shaped and rule-governed behavior: Instructional control of human loss avoidance. Journal of the Experimental Analysis of Behavior, 31, 53-70. doi:10.1901/jeab.1979.31-53

Glenn, S. S. (1986). Metacontingencies in Walden Two. Behavior Analysis and Social Action, 5, 2-8.

Hayes, S. C., Brownstein, A. J., Zettle, R. D., Rosenfarb, I., \& Korn, Z. (1986). Rule governed behavior and sensitivity to changing consequences of responding. Journal of the Experimental Analysis of Behavior, 45, 237-256. doi:10.1901/ jeab.1986.45-237

Hanna, E. S., \& Todorov, J. C. (2002). Modelos de autocontrole na análise experimental do comportamento: Utilidade e crítica. Psicologia: Teoria e Pesquisa, 18, 337-343. doi:10.1590/ S0102-37722002000300014

Hoerger, M. L., \& Mace, F. C. (2006). A computerized test of selfcontrol predicts classroom behavior. Journal of Applied Behavior Analysis, 39, 147-159. doi:10.1901/jaba.2006.171-04 
Gonçalves, A. S., Albuquerque, L. C. \& Paracampo, C. C. P. (2015). Controle por Regras: Efeitos de Perguntas, Propriedades Formais de Regras e Esquemas de Reforço.

La Piedad, X., Field, D., \& Rachlin, R. (2006). The influence of prior choices on current choice. Journal of the Experimental Analysis of Behavior, 85, 3-21. doi:10.1901/jeab.2006.132-04

LeFrancois, J. R., Chase, P. N., \& Joyce, J. (1988). The effects of variety of instructions on human fixed-interval performance. Journal of the Experimental Analysis of Behavior, 49, 383393. doi:10.1901/jeab.1988.49-383

Martinez, H., \& Tomayo, R. (2005). Interactions of contingencies, instructional accuracy, and instructional history in conditional discrimination. The Psychological Record, 55, 633-646.

Martone, R. C., \& Todorov, J. C. (2007). O desenvolvimento do conceito de metacontingência. Revista Brasileira de Análise do Comportamento, 3, 181-190.

Najjar, E. C. A., Albuquerque, L. C., Ferreira, E. A. P., \& Paracampo, C. C. P. (2014). Efeitos de regras sobre relatos de comportamentos de cuidados com os pés em pessoas com diabetes. Psicologia: Reflexão e Critica. doi:10.1590/16787153.201427215

Newman, B., Buffington, D. M., \& Hemmes, N. S. (1995). The effects of schedules of reinforcement on instruction following. The Psychological Record, 45, 463-476.

Oliveira, V. L., \& Albuquerque, L. C. (2007). Efeitos de histórias experimentais e de esquemas de reforço sobre o seguir regras. Psicologia: Teoria e Pesquisa, 23, 217-228. doi:10.1590/ S0102-37722007000200012

Oliveira-Castro, J. M. (2003). Effects of base price upon search behavior of consumers in a supermarket: An operant analysis. Journal of Economic Psychology, 24(5), 637-652. doi:10.1016/S0167-4870(03)00006-0

Okoughi, H. (1999). Instructions as discriminative stimuli. Journal of the Experimental Analysis of Behavior, 72, 205-214. doi:10.1901/jeab.1999.72-205

Paracampo, C. C. P., \& Albuquerque, L. C. (2004). Análise do papel das consequências programadas no seguimento de regras. Interação em Psicologia, 8, 237-245. doi:10.5380/ psi.v8i2.3259

Paracampo, C. C. P., Albuquerque, L. C., Farias, A. F., Carvalló, B. N., \& Pinto, A. R. (2007). Efeitos de consequências programadas sobre o comportamento de seguir regras. Interação em Psicologia, 11, 161-173. doi:10.5380/psi.v11i2.7850

Paracampo, C. C. P., Albuquerque, L. C., Mescouto, W. A., \& Farias, A. F. (2013). Efeitos de perguntas e de respostas às perguntas sobre o seguir regras. Psicologia: Teoria e Pesquisa 29(4), 369-379.

Paracampo, C. C. P., Souza, L. M., \& Albuquerque, L. C. (2014). Variáveis que podem interferir no seguir regras de participantes flexíveis e inflexíveis. Psicologia: Reflexão e Crítica, 27(1), 124-133. doi:10.1590/S0102-79722014000100014

Perez, W. F., Reis, M. J. D., \& de Souza, D. G. (2009). Efeitos de história experimental com diferentes instruções e do controle por contingências sobre o seguimento de instruções. Acta Comportamentalia, 18, 55-85.

Schlinger, H., \& Blakely, E. (1987). Function-altering effects of contingency-specifying stimuli. The Behavior Analyst, 10, 41-45.

Skinner, B. F. (1969). Contingencies of reinforcement: A theoretical analysis. New York: Appleton-Century-Crofts.

Skinner, B. F. (1974). About behaviorism. New York: Alfred A. Knopf.

Torgrud, L. J., \& Holborn, S. W. (1990). The effects of verbal performance descriptions on nonverbal operant responding. Journal of the Experimental Analysis of Behavior, 54, 273 291. doi:10.1901/jeab.1990.54-273
Wulfert, E., Greenway, D. E., Farkas, P., Hayes, E. C., \& Douguer, M. J. (1994). Correlation between self-reported rigidity and rule-governed insensitivity to operant contingencies. Journal of Applied Behavior Analysis, 27, 659-671. doi:10.1901/ jaba.1994.27-659.

Zettle, R. D., \& Hayes, S. C. (1982). Rule-governed behavior: A potential theoretical framework for cognitive-behavior therapy. In P. C. Kendall (Ed.), Advances in cognitivebehavioral research and therapy (pp. 73-118). New York: Academic Press. 